Dr. med. Johannes G. Schmidt, Einsiedeln

\section{Chronische Erkrankungen und Multimorbidität}

Was sind chronische Krankheiten? Ein Mensch wird krank, wenn er gesund reagiert, und er bleibt (scheinbar) gesund, wenn er krankhaft reagiert. So führt etwa das Eindringen einer Grippe zu Gliederschmerzen, Fieber, Schwitzen, Katarrh und Krankheitsgefühl, und durch diese gesunde Reaktion kann ein Mensch die eingedrungene Kälte wieder eliminieren. Er wird vorübergehend krank, und daraus resultiert eine restitutio ad integrum die Gesundung. Kann ein Mensch aufgrund angeborener oder erworbener Schwächen nicht gesund reagieren (oder nimmt er sich keine Zeit dazu), dann spürt er von der eingedrungenen Grippe nichts und bleibt scheinbar gesund - doch jetzt fehlt die gesundheitliche Integrität und die eingedrungene Kälte beginnt, kleine und mit der Zeit auch grössere Störungen zu verursachen. Es entsteht der Boden für eine chronische Krankheit. Epidemiologisch entscheidend ist das Zusammenwirken von Krankheit und Wirt. So sind es auch der Wirt und sein Gesundungsvermögen, die bestimmen, wie eine Krankheit verläuft und ob sie chronisch wird.

Multimorbidität ist ein Ausdruck davon, dass ein Patient nicht mehr mag und seine Resistenz verloren hat. Multimorbidität ist ein Symptom des Wirts. Sie ist nicht einfach die Addition mehrerer Krankheiten, sondern eine Diagnose über den Wirt - ein Zeichen der erschöpften Widerstands- und Gesundungskräfte des ganzen Patienten. Wenn es gelingt, eine Krankheit erfolgreich zu kontrollieren, wissen wir noch nicht, ob der ganze Mensch effektiv gesünder geworden ist. Eine echte restitutio kann eigentlich erst entstehen durch eine Gesundung derjenigen zentralen Funktionen, die eine Regeneration der Kräfte und der Beherztheit erlauben und so das Wehrvermögen des Körpers wiederherstellen. In erster Linie sind dies gesunder Schlaf, gute und geformte Verdauung und innere Wärme mit warmen Füssen. Gelingt eine Stärkung des Wirts und seines Gesundungsvermögens, kommt es oft zu einer zeitweisen Verstärkung oder Rückkehr der Krankheiten und der Beschwerden. Der erstarkte Organismus will diese überwinden und eliminieren und möchte sie nicht nur ruhiggestellt haben. Der erstarkte Wille zur Gesundung lässt Schmerzen und Beschwerden auch besser ertragen und aushalten, sodass der salutogene Aufräumprozess dann (ein Stück weit) gelingen kann.

In der Tat wissen wir bei erfolgreichen Therapien von Krankheiten heute allermeist nicht, ob sich dabei auch die Gesamtmorbidität und Gesamtmortalität verbessern. Und wo diese in grossen kontrollierten Studien erfasst worden sind, sehen wir meistens keine Veränderung, sodass eine erfolgreiche Krankheitsbehandlung nicht unbedingt den ganzen Menschen als Wirt gesunden lässt, sondern vielfach nur dessen Krankheiten und Todesursachen austauscht. Die klinische Epidemiologie als wissenschaftliche Basis der modernen Medizin möchte alle relevanten Determinanten von Krankheit und Wirt erhellen und deren jeweilige Relevanz auf das Krankheitsgeschehen bestimmen. Schon lange wird deshalb eine Klassifikation des Wirts postuliert, weil - unübersehbar - Krankheiten je nach Patient völlig unterschiedlich verlaufen. Zwischen der real existierenden medizinischen Praxis, die im Falle von Multimorbidität ganze Diagnoselisten aufstellt und für alle Einzeldiagnosen eine entsprechende Therapie appliziert und so auch ganze Therapielisten erstellt, zwischen dieser disputablen Praxis und den systematischen wissenschaftlichen Erkenntnissen, die sich etwa in der Cochrane Library sammeln, besteht heute eine grosse Diskrepanz. So hat z.B. die Statin-Behandlung, die heute angeblich jede und jeder mit einem hohen Cholesterin haben muss, keine eindeutige präventive Wirkung, wo nicht schon ein Infarkt vorliegt. Ein Wirt ohne Infarkt weist also ein ausreichendes Gesundungsvermögen auf, um mit der pathogenen Wirkung hoher Cholesterinwerte zurechtzukommen. Erst ein Infarkt gibt einen genügenden Hinweis darauf, dass der betroffene Wirt ein Problem mit dem Cholesterin hat (und das auch bei normalen Werten).

Es ist immer ein und derselbe Mensch, der «multimorbid» ist und mehrere Krankheiten hat. Der Wirt ist somit der gemeinsame Nenner für multiple Krankheiten. Ein Beispiel: Schwere chronisch obstruktive Lungenkrankheit (COPD), Panikattacken und Angstsymptomatik bei

\section{KARGER}

Fax +497614520714 Information@Karger.de www.karger.com $1015-0684 / 12 / 0246-0324 \$ 38.00 / 0$
Dr. med. Johannes G. Schmidt

Stiftung Paracelsus heute

Praxiszentrum Meinradsberg

Ilgenweidstrasse 3, 8840 Einsiedeln, Schweiz

schmidt@paracelsus-heute.ch 
histrionischer Persönlichkeitsstörung, Nikotinabusus, Steatosis hepatis, gestörte Glukosetoleranz unter Prednisontherapie, (nicht operiertes) Mammakarzinom bei Status nach jahrelanger Hormontherapie, Aortensklerose, Status nach Appendektomie, Status nach Konisation usw. Also: Ein Wirt mit Mitte-Schwäche seit der Geburt, LeberQi-Stagnation mit gestörter Leber-Lungen-Balance, Erschöpfung der Nierenenergie und Yang-Mangel. Entsprechend muss die therapeutische Strategie zur Stützung des Gesundungsvermögens sein: Aufbau der Mitte und Regulierung der Lungen-Leber-Dysbalance, wobei auch inhalative Antiasthmatika und Sauerstoff (vorläufig) nötig sind. Weil das Rauchen die kalte Lunge wärmt und so unterstützt, ist ein Nikotinstopp vorerst keine gangbare Option. Auch für eine Resektion des Mammakarzinoms sind die Voraussetzungen vorläufig nicht gut, weil der Wirt noch zu schwach ist. Es ist ja auch nicht das (stabile) Mammakarzinom, sondern die schwere Lungenkrankheit, welche die Lebenserwartung limitiert. Die 60-jährige Patientin hat seit der Behandlung mittels Altchinesischer Medizin keine Hospitalisationen und auch keine Antibiotika und Prednison-Gaben mehr benötigt. Krisenhafte Phasen sind wiederholt vorgekommen, weil die schwache Mitte das Gleichgewicht nicht halten kann, das durch vielfältige Einflüsse immer wieder an den Rand gebracht wird: durch Wind und Wetter, die Tagesenergie, Wechsel in der Natur, kraftlose Ernährung, Sorgen usw. Der wichtigste Erfolgsindikator ist die Äusserung der Patientin, dass sie sich nach 2 Jahren Therapie selber besser zu spüren beginnt und mehr «zu sich gekommen» ist (nachdem massenhaft Schleim gelöst worden ist und weiter gelöst wird). Und trotz zeitweise grossen Ängsten und heftigen Leidensphasen sind Schlaf und Appetit nie eingebrochen. Um die therapeutische Orientierung zu behalten, gilt es, ganz die Entwicklung der Kräfte im Auge zu behalten, wobei Zungenund Pulsbefund immer wieder hilfreich sind. Und es braucht eine strategische Sicht auf einen Verlauf, der sich nur langsam und über mehrere Jahre hinweg verbessern kann.

Für die Restitution des Wirts, der (multiple) chronische Krankheiten überwinden will, sind fast immer Verfahren, Arzneien und eine Ernährung nötig, die Bauch und Niere wärmen und stärken und es ums Herz warm werden lassen. Und Vorsicht: Entzündungen und Hitze sind fast immer «falsche Hitze», die auf dem Boden von innerer Kälte und Yang-Mangel (fehlende «Lebenswärme») entsteht. Werden (entzündliche) Krankheiten ohne Bewahrung des Yang therapeutisch «kaltgestellt», was unglücklicherweise auch viele Komplementärmediziner heute tun, dann geht dies auf Kosten der Vitalität und des Gesundungsvermögens. Um richtig gesund zu werden, braucht der Körper seine Vitalität, um Krankheitskrisen hervorrufen zu können, wo diese einen Wehrvorgang und Aufräumprozess darstellen. So lässt sich gut verstehen, dass chronische Krankheiten heute auch die Folge iatrogener Einflüsse durch eine Medizin sind, die zwar gelegentlich erfolgreich Einzelkrankheiten eindämmen kann, aber keine Klassifikation und Lehre des Wirts und seiner Gesundheit und Gesundung kennt - und übersieht, dass ihr meist eine Wirkung auf die Morbidität und Mortalität insgesamt fehlt. Das fehlende Verständnis von Gesundheit und salutogener Prozesse in der heutigen Medizin - diese grosse Lücke zu schliessen, scheint mir die Aufgabe und Bestimmung der Komplementärmedizin zu sein. 